\title{
The solar cycle as a forced and damped harmonic oscillator: long-term variations of the amplitudes, frequencies and phases
}

\author{
K. M. Hiremath
}

\author{
Indian Institute of Astrophysics, Bangalore-560034, India \\ e-mail: hiremath@iiap.res.in
}

Received 28 December 2004 / Accepted 24 October 2005

\begin{abstract}
We model solar activity cycle as a forced and damped harmonic oscillator consisting of two parts, sinusoidal and transient. The amplitudes, frequencies, phases and decay factors of such a harmonic oscillator are determined by fitting the equation of the sinusoidal and transient parts to the sunspot data for the years 1755-1996 (cycles 1-22) with the results: (i) there is a long-term decreasing trend in the phase, while the amplitude and the frequency (or period of $\sim 11 \mathrm{yr}$ ) of the sinusoidal part remain constant for all the solar cycles; (ii) the amplitude of the transient part is phase locked with the phase of the sinusoidal part; (iii) for all the cycles, the period and decay factor (that is much less than 1) of the transient part remain approximately constant; (iv) except in cycles 6 and 15 , the phases of the transient part are approximately constant with a magnitude of $\sim \pi / 2$ radians and; (v) for cycles 6 and 15 , the simultaneous change in magnitude of phase difference ( $\sim 2 \pi$ radians) between the transient and sinusoidal parts and of very low sunspot activity may be due to the Maunder minimum type of oscillations.

The constancy of the amplitudes and the frequencies of the sinusoidal part and a very small decay factor from the transient part suggests that the solar activity cycle mainly consists of a persistent oscillatory part that might be compatible with long-period ( $22 \mathrm{yr})$ Alfven oscillations.
\end{abstract}

Key words. sunspots - Sun: activity - Sun: interior - Sun: oscillations

\section{Introduction}

Since the discovery of sunspots by Galileo and their 11 year cyclic activity by Schwabe, the physics of their origin and phenomena of their cyclic activity have not yet been understood completely. The persistence of the 11 year solar cycle is often debated in the literature. From the analysis of available sunspot data, most of the previous studies demonstrate the presence of the 11 year sunspot activity or 22 year magnetic activity. Using different mathematical tools, several studies show that the solar activity is a regular phenomenon and not a chaotic one. Dicke's (1978) analysis of sunspots and solar-terrestrial weather indicated a regular clock inside the sun. The 22 year magnetic activity can be due to long period Alfvenic perturbations of the underlying magnetic field structure in the solar interior that may be of primordial origin. The importance of such a fossil field on the dynamo generated magnetic field in the convective envelope was examined by Boyer \& Levy (1984). Long-term solar activity and prolonged periods of low solar activity can be explained (Pudovkin \& Benevolensky 1985) by taking into account the effect of a quasi-stationary internal magnetic field.

Since the ancient varves' records are influenced by the changing earth's climate due to the influence of the sun, analysis of the varved rocks show a persistent $22 \mathrm{yr}$ solar effect (Sonett \& Trebisky 1986). From the Hurlst analysis of $C^{14}$ data of $6000 \mathrm{BC}$ - 1950 AD, Ruzmaikin et al. (1994) found the persistence of the long-term solar activity. Hurlst analysis of rotation rates parameters (A, B and C) measured from the Mount Wilson Doppler velocity data shows a persistent behavior of the solar activity on time scales of 20 days to a few thousand years (Komm 1995). Polygiannakis et al. (1996) developed a model based on a nonlinear RLC circuit that yields physical characteristics of the solar cycle and show the Maunder minimum type behavior of the solar cycle. A Fourier spherical harmonic analysis of the magnetic field (Stenflo \& Vogel 1986; Knaack \& Stenflo 2005) and of the field inferred from the sunspot groups (Gokhale et al. 1989; Gokhale \& Javaraiah 1990) show the dominant 22 year periodicity. Recent analysis (Mursula et al. 2001) of the group sunspot number (GSN) series of Hoyt \& Shatten (1998) shows persistent 22 year periodicity through out the 400 years of sunspot activity that includes the Maunder minimum period. Compared to the Wolf sunspot number series, the GSN series includes all the known archival records of sunspots that include the observations by Galilei in 1610. Most of these studies strongly suggest that the solar cycle and activity phenomena may originate in the long period ( $\sim 22 \mathrm{yr})$ Alfven perturbations of magnetic field structure in the solar interior (see for example Hiremath \& Gokhale 1995). Verdes et al. (2004) considered long-term solar magnetic activity as a nonstationary time series that incorporates secular changes into the modeling process through an external driving parameter. Compared to standard stationary methods, their method predicts the characteristics of the solar cycle very well.

Most of the studies that search for periodicities in sunspot activity assume that the solar cycle is a sinusoidal variable. Although the rising part (minimum to maximum) of the curve of the solar activity cycle appears to be sinusoidal, its declining part (maximum to minimum) shows behavior similar to that of a damped harmonic oscillator (see the Fig. 14 of 
Tipler \& Moska 2003). Thus solar cycle amplitude variations can be described by the combined solutions of a natural (sinusoidal) and a damped harmonic oscillator. However such natural oscillations of $22 \mathrm{yr}$ periodicity that excite in the solar interior, while traveling towards the surface, ultimately must be damped by strong dissipation in the convective envelope. Hence, in order to re-excite and maintain 22 year oscillations, a persistent perturbation (of the poloidal magnetic field structure of primordial origin that pervades the solar interior) is necessary.

Thus keeping in mind that shape of the sunspot activity cycle may be described as a forced and damped harmonic oscillator, using long-term series (1755-1996) of sunspot data, we test our proposition and present the amplitudes, phases and decay constants for each solar cycle. In Sect. 2, we present our model of the solar cycle as a forced and damped harmonic oscillator. The data and results are presented in Sect. 3 and, conclusions are presented in Sect. 4.

\section{Forced and damped harmonic oscillator}

In order to understand the physics of the solar cycle, we make the assumption that the shape of the solar cycle can be described by the combined solution of a forced and damped harmonic oscillator. From any standard physics text book (ex., Tipler \& Mosca 2003), one obtains the following solution for such a non-linear harmonic oscillator:

$y=A_{1} \cos \left(\omega t-\phi_{1}\right)+A_{2} \cos \left(\omega^{\prime} t-\phi_{2}\right) \mathrm{e}^{-\gamma t}$,

where $y$ is displacement (in the present context we consider the Wolf sunspot number), $A_{1}, A_{2}$ are amplitudes, $\omega(2 \pi / T$, where $T$ is the period in years) is the sinusoidal frequency, $\omega^{\prime}\left(2 \pi / T^{\prime}\right.$, where $T^{\prime}$ is the period in years) is the damping frequency, $\phi_{1}$ and $\phi_{2}$ are the phases, $\gamma$ is the decay factor and $t$ is the time variable in months. Note that the first term in the above equation is the steady-state solution whose natural oscillations are maintained by an external force and the second term is related to the transient solution dictated by the damping of the system.

\section{Data and results}

For the present study, we consider the monthly means of the Wolf sunspot numbers. For the years 1755-1960 we consider the data collected by Waldmeier (1961) and for the years 1961-1996 we consider the data collected from Solar Geophysical Data (USA). For the period 1755-1996, in the upper part of Fig. 1, the monthly Wolf number means (hereafter called the monthly mean) are presented. In the lower part of Fig. 1, we illustrate the number of data points in each solar cycle. For example, the period of the solar cycle 1 (1755-1765) is 11 years (or 132 months) and thus we get 132 monthly mean values. Similarly the period of solar cycle 2 (1766-1774) is 9 years (108 months) and we get 108 monthly mean values of the sunspot activity and so on.

Since the monthly mean of the sunspot data is noisy, before fitting the data to Eq. (1), we smooth each set of data by computing the 12 data points running mean. For example, if the data consists of $n$ data points and $j$ is the number of data points used to compute the running mean, then we have $n-j$ smoothened data points. Thus for cycle 1 , we have 120 smoothened monthly means. After smoothing, for each cycle, we compute the average of the smoothened monthly means. We call this average of the smoothened monthly mean as the cycle mean of the sunspot activity. For each cycle of the smoothened monthly mean, using the
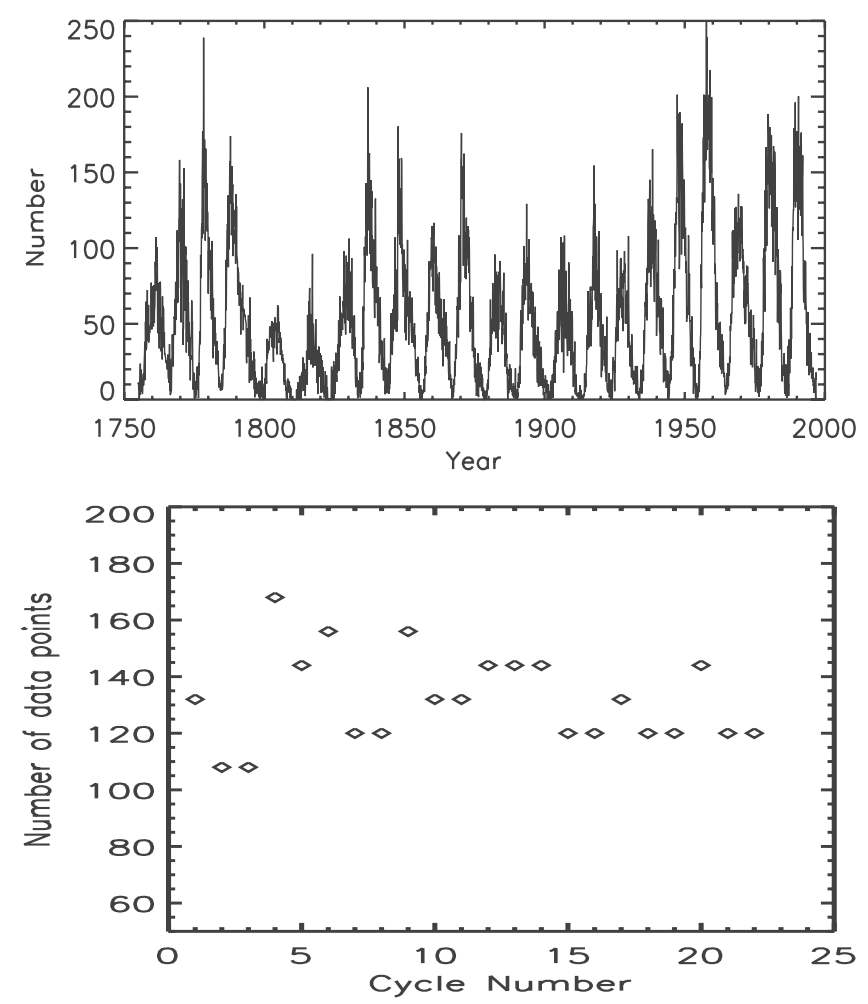

Fig. 1. The upper figure illustrates the monthly mean of the Wolf sunspot number for the period 1755-1996 and the lower figure illustrates the number (before smoothening) of data points for the cycles 1-22.

cycle mean, we compute the standard deviation. Then we compute the normalized deviation data as follows. First we compute the deviation of the cycle mean from the smoothened monthly mean and then the resulting data is normalized by the standard deviation. Thus, if $x_{i}$ are the smoothened monthly means for different $i$ months, $\bar{x}$ is the cycle mean of all the data points and $\sigma$ is the standard deviation, then the normalized deviation of the sunspot data is represented by $y_{i}=\left(x_{i}-\bar{x}\right) / \sigma$. For the year 1987-1996, the normalized deviation of the smoothened monthly means of the sunspot data is illustrated in Fig. 2a.

For all the 22 cycles, the normalized deviation data is subjected to a non-linear least square fit by the LevenbergMarquardt method (Press et al. 1992), which is an iteration process that derives unknown coefficients (like $A_{1}, \phi_{1}$, etc.), from an initial reasonable guess of the coefficients. In order to have a fast convergence of the iteration process, we adopt the following method. By neglecting the second, transient part of Eq. (1), we determine the coefficients $A_{1}, \omega, \phi_{1}$ from the linear least square fit of the normalized deviation data. We assume that the coefficients $\left(A_{2}, \omega^{\prime}, \phi_{2}\right.$ and $\left.\gamma\right)$ of the transient part of Eq. (1) are equal to the values of coefficients $A_{1}, \omega, \phi_{1}$ and $\gamma=1$. Using these coefficients, the iteration process leads to fast convergence and finally we get the correct values of the unknown coefficients in Eq. (1). We compute a non-linear least square fit (the Levenberg Marquardt algorithm) using standard IDL software and obtain different parameters (with their uncertainties) and as well as the value of $\chi^{2}$.

To test the goodness of the fit, we compute the value of $\chi^{2}$. Since the measurement errors are not available for the monthly sunspot data, according to IDL software, we assume the square root of sunspot numbers as the measurement errors. In this way, we fit the sunspot data of each solar cycle (from 1-22) to Eq. (1) 

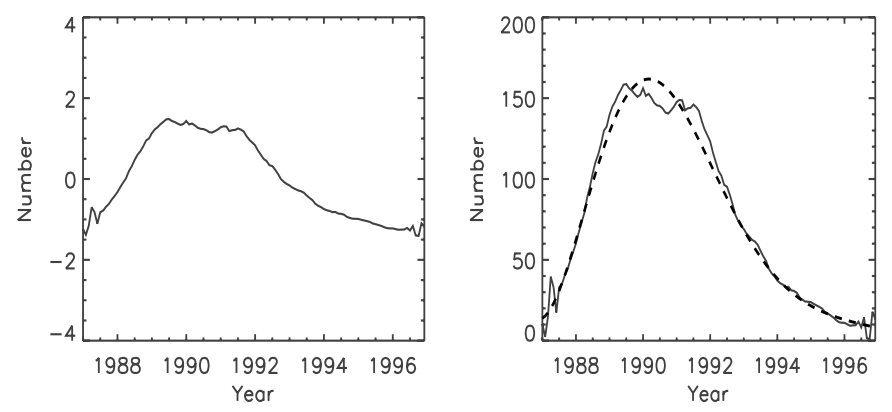

Fig. 2. a) The left figure illustrates the normalized deviation of smoothed monthly mean. b) The right figure illustrates the monthly mean. The continuous line is the observed monthly mean and the dashed line is obtained from the non-linear least square fit to Eq. (1) of the forced and damped harmonic oscillator.

and obtain the amplitudes, the frequencies and the phases of the forced and damped harmonic oscillator. For the sunspot cycle 1987-1996, a typical non-linear least square fit of Eq. (1) to the sunspot data is presented in Fig. $2 b$ (dashed line).

In Fig. 3, we present the parameters (and their uncertainties) of the sinusoidal part (the first term on the right hand side of the Eq. (1)). In Fig. 3 for all the solar cycles, the amplitudes and the frequencies remain almost constant except for a decrease in phase as cycle number increases. In fact the mean frequency of such a forced oscillator for all the solar cycles is $\sim 0.55$ (or a period of $\sim 11 \mathrm{yr}$ ). Using the group sunspot numbers of Hoyt \& Shatten (1998), on average, the same 11 yr cycle period was obtained in the previous studies (Hathaway et al. 2002; Hathaway \& Wilson 2004).

In Fig. 4, we present the parameters (and their uncertainties) of the transient part (the second term on the right hand side of Eq. (1)). Both amplitudes of the sinusoidal and transient parts are negative. This is due to the fact that for all the solar cycles data, we obtain nearly $67 \%$ of the normalized deviation values as negative; the rest are positive. Thus derived amplitudes of both the sinusoidal and transient parts of Eq. (1) are weighted towards large numbers of negative values. The typical deviation of such data is presented in Fig. 2a. Using the cycle mean $\bar{x}$ and standard deviation $\sigma$, one can transform the normalized deviation data into monthly mean data (the continuous curve in Fig. 2b; in the same figure the dashed line superposed on the continuous line is obtained from the non-linear least square fit). In Fig. 5a, we present the observed (the difference between the maximum and the minimum of number of sunspots) and computed amplitudes. The amplitudes obtained from the fit are similar to the observed amplitudes.

For each solar cycle, we have at least 120 monthly means of the sunspot data. When we smoothed each solar cycle data with a 12 point running mean, we have at least 108 data points in each cycle and with the 7 unknown parameters, the degrees of freedom for each value of $\chi^{2}$ is $n-7$ (where $n$ is the number of smoothed data points) yielding 101 degrees of freedom. For comparison, in the right part of Fig. 4 we plot values of the $\chi^{2}$ (dash with three dotted line). In Fig. 4b the maximum value of $\chi^{2}$ is $\sim 10$ and thus the reduced value $\chi_{v}^{2}$ is $\sim 10 / 101$ $(\sim 0.1)$. From standard statistical tables (see for example Table C4 in "Data reduction and error analysis for the physical sciences", by Bevington \& Robinson 1969), the significance of the $\chi^{2}$ is $>99 \%$ yielding that for all the solar cycles $\chi^{2}$ fits have very high significance.

The results for the damped (transient) part of the oscillator are: (i) the frequencies $\omega^{\prime}$ remain approximately constant and

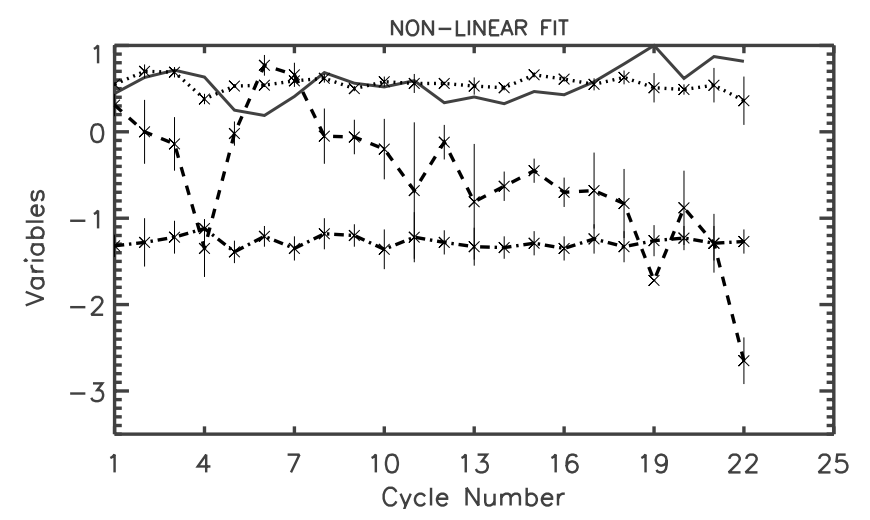

Fig. 3. For solar cycles 1-22 (1755-1996), the coefficients obtained from the non-linear least square fit of sunspot data to the first term (sinusoidal part) of Eq. (1) are shown. The continuous line is the cycle mean (normalized to the maximum value in all the solar cycles). The dash with dotted line is the amplitude $A_{1}$, the dotted line is the frequency $\omega$ and the dashed line is the phase $\phi_{1}$.
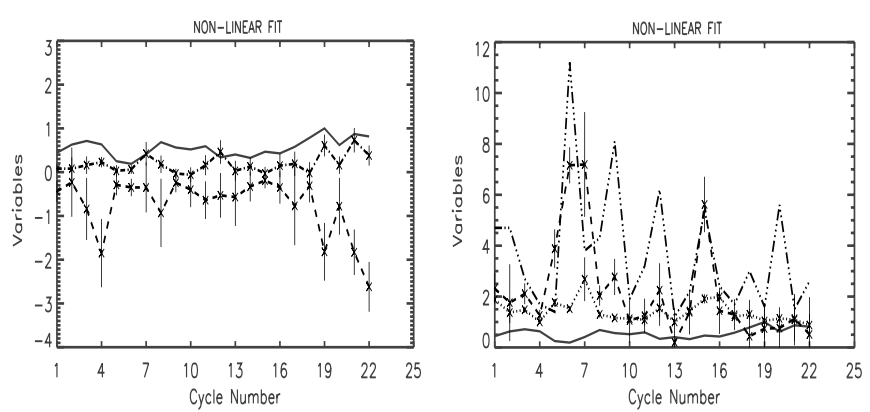

Fig. 4. For solar cycles 1-22 (1755-1996), the coefficients obtained from the non-linear least square fit of sunspot data to the second term (transient part) of Eq. (1). In both the plots the continuous line is the cycle mean (normalized to the maximum number in all the solar cycles). a) In the left figure, the dashed line is the amplitude $A_{2}$ and the dash with dotted line represents the decay factor $\gamma$ respectively. b) In the right figure, the dotted line is the frequency $\omega^{\prime}$ and the dashed line is the phase $\phi_{2}$. The dash with three dotted line represents the values of $\chi^{2}$ for each cycle.

are in the range of 1-1.5 (or a period of 5-6 years); (ii) the amplitude $A_{2}$ is phase locked with the phase of the sinusoidal part of the forced oscillator (this conclusion is based on the result of a significant correlation (64\%) between $A_{2}$ and $\phi_{1}$ ); (iii) except during the cycles 6 and 15 (when the phase reaches its maximum value of $\sim 2 \pi$ radians), the phase remains almost constant and is on average $\sim \pi / 2$ radians; (iv) the decay factor that determines the shape of the latter part of the solar cycle is approximately constant and is much less than 1; and (v) for the cycles 6 and 15, the change of magnitude of the phase (from the minimum values to $2 \pi$ radians) $\phi_{2}$ occurs and correspondingly the solar cycles have very low sunspot activity.

The present analysis also shows negative phases for the sinusoidal part and positive phases for the transient part which might indicate different arrival times of waves towards the surface. As already outlined, except for cycles 6 and 15, the average phase of the transient part is $\sim \pi / 2$ radians. Thus except for cycles 6 and 15, the transient part can be represented as $A_{2} \cos \left(\omega^{\prime} t-\pi / 2\right) \mathrm{e}^{-\gamma t} \sim A_{2} \sin \left(\omega^{\prime} t\right) \mathrm{e}^{-\gamma t}$ and the Eq. (1) is modified into the following form:

$y \approx A_{1} \cos \left(\omega t-\phi_{1}\right)+A_{2} \sin \left(\omega^{\prime} t\right) \mathrm{e}^{-\gamma t}$. 

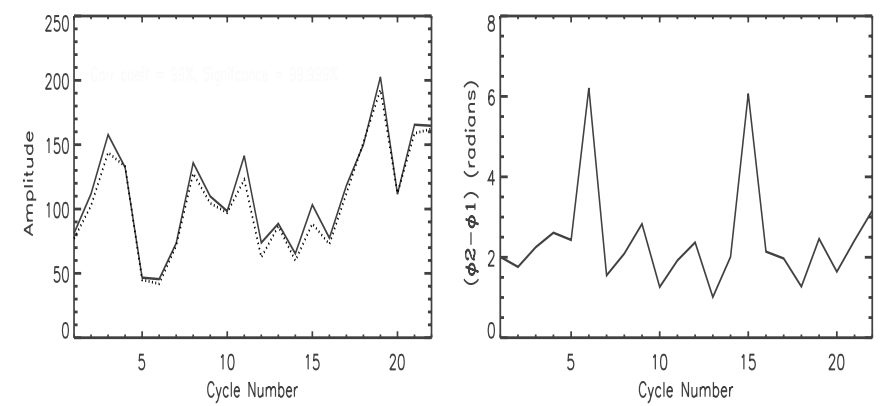

Fig. 5. a) The left figure illustrates the comparison between the amplitudes of the observed sunspot cycle and the amplitudes obtained from the non-linear least square fit of Eq. (1) of a forced and damped harmonic oscillator. The continuous line is the observed sunspot amplitudes obtained by smoothing the raw data. The dotted line shows the amplitudes obtained from the non-linear least square fit. The correlation between the two variables is found to be $99 \%$ with high significance. b) The right figure represents the phase difference $\left(\phi_{2}-\phi_{1}\right)$ between the transient and the sinusoidal parts of a forced and damped harmonic oscillator.

Thus there is a phase lag between the transient part and sinusoidal part of the forced and damped harmonic oscillator. The phase difference $\left(\phi_{2}-\phi_{1}\right)$ between the transient and sinusoidal parts is illustrated in Fig. 5b. Note the gradual changes in the phase difference ( $\sim 2 \pi$ radians) during the cycles 6 and 15 .

In order to investigate whether the result (v) concerning cycles 6 and 15 might be interpreted as a signature of the 80 year Gleissberg cycle or as due to a Maunder minimum type of oscillation, we performed the FFT analysis for the detection of the periodicity followed by the wavelet analysis. To obtain the FFT and wavelet power spectra, we adopted the following procedure. First we compute the overall mean and standard deviation (This overall mean and standard deviation is for the $242 \mathrm{yr}$ and should not be confused with the cycle mean and standard deviation) from the monthly means of the sunspot data. Then we compute the normalized deviation (similar to method of the normalized deviation of monthly means) of the 242 year data. In Fig. 6, we present the power spectra of both the FFT (upper figure) and wavelet (lower figure) analyses. Note that the 11 year cycle is the only dominant periodicity in both FFT and wavelet power spectra. The power concentrated in the hatched region of the wavelet power spectrum is the result of adding zeros to the data set before computing the wavelet spectrum and is not considered to be significant (see the method of obtaining the wavelet transform at the website: http: //paos.colorado.edu/research/wavelets/ developed by Torrence and Compo). Thus one has to consider the concentrations of the power above the hatched region as real periods. Although there appears to be a $100 \mathrm{yr}$ periodicity in the low frequency side of the FFT analysis, in the wavelet analysis the $80-100 \mathrm{yr}$ period is not as regular as the $11 \mathrm{yr}$ period and moreover most of the power in the range of the 80-100 yr periodicity is concentrated in the hatched region which is statistically not significant. Thus with the present data set we cannot unambiguously state that there is an 80 year Gleissberg cycle. Previous studies also show that an 80 year Gleissberg cycle need not be 80 year long and may be a cycle of 100 years (Rozelot 1994; Bonev et al. 2004).

We can further justify our conjecture that the gradual change of phase of $2 \pi$ radians between the transient and sinusoidal parts leads to a Maunder minimum type of oscillations as follows. If these 100 year Maunder minimum type of oscillations are real
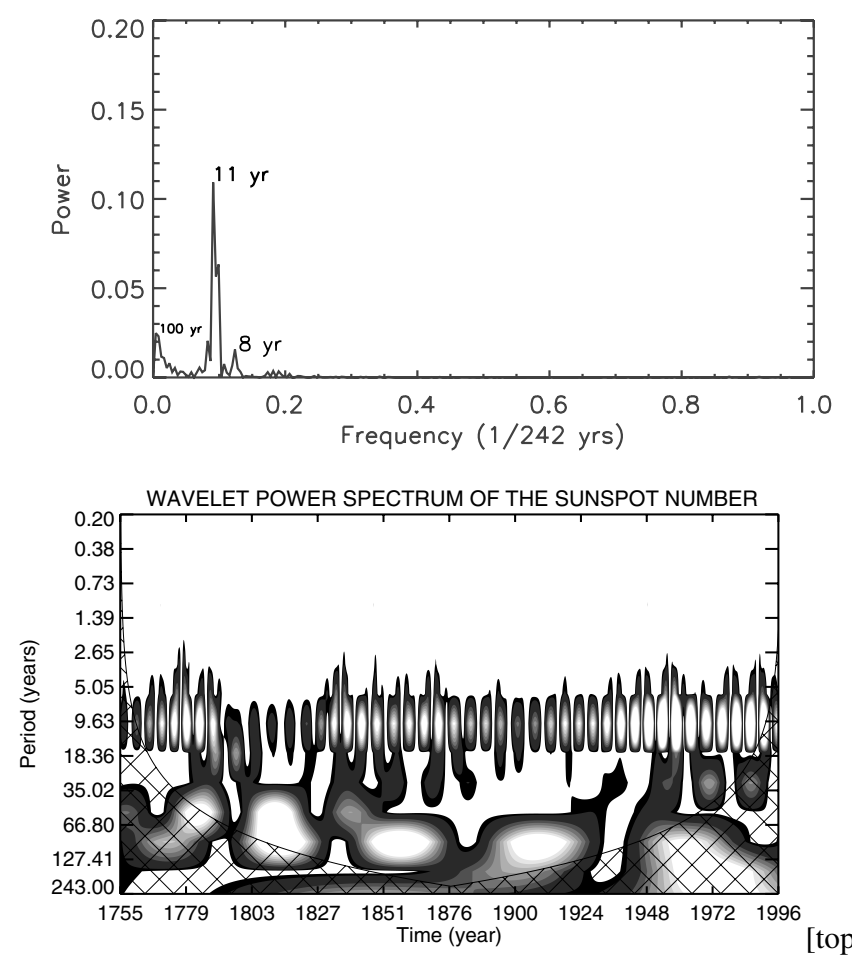

Fig. 6. Power spectrum analysis of the monthly mean of the sunspot data. a) The upper figure represents the FFT power spectrum, b) the lower figure represents the wavelet power spectrum. The detected periods from the FFT analysis have powers greater than the $3 \sigma$ level.

(that is, around 1810-1822 and 1914-1923 the sunspot activity is low), then we should expect that in the past (around the years $1710-1722,1610-1622,1510-1522,1410-1422,1310-$ $1322,1210-1222,1110-1122,1010-1022,910-922,810-822$ and so on) the sunspot activity was low. When we checked these years from the reconstructed sunspot data (Usoskin et al. 2004, see their Figs. 5 and 6) surprisingly around these years we get very low sunspot activity. Moreover the FFT analysis of the reconstructed sunspot data by the same authors shows a statistically significant period of 100 years. However, we have to further verify the validity of our conjecture by using reconstructed sunspot data and using the methods presented in this study.

\section{Conclusions}

The conclusions of the present study are: (i) the solar cycle can be described as a forced and damped harmonic oscillator, (ii) there is a long-term decreasing trend in the phase, while the amplitude and the frequency of the sinusoidal part of the forced oscillations are almost constant for all 22 solar cycles, (iii) except during cycles 6 and 15, the phases of the transient part remain approximately constant and are $\sim \pi / 2$ radians; (iv) the amplitude of the transient part is phase locked with the phase of the sinusoidal part of the forced oscillations, (v) the decay factor $\gamma$ in each solar cycle is negligible $(<1)$ and remains constant for all 22 solar cycles; (vi) simultaneous change in magnitude of the phase difference ( $\sim 2 \pi$ radians) between the transient and sinusoidal parts of cycles 6 and 15 that of very low sunspot activity may be due to the Maunder minimum type of oscillations and; (vii) constancy of the amplitudes and frequencies of the sinusoidal part and very low dissipation $(\gamma<1)$ of the damping oscillator indicate that the solar activity cycle might be described 
by persistent MHD oscillations due to long-period ( $22 \mathrm{yr}$ magnetic activity) Alfvenic perturbations.

Acknowledgements. The author is grateful to the anonymous referee for many helpful and insightful comments that contributed to the significant improvement of the manuscript. We used the wavelet software developed by $C$. Torrence and $G$. Compo that is available at the web site http://paos.colorado.edu/research/wavelets/

\section{References}

Bonev, P. P., Penev, K. M., \& Sello, S. 2004, ApJ, 605, L81

Boyer, D., \& Levy, E. H. 1984, ApJ, 277, 848

Dicke, R. H. 1978, Nature, 276, 676

Gokhale, M. H., \& Javaraiah, J. 1990, MNRAS, 243, 241

Gokhale, M. H., Javaraiah, J., \& Hiremath, K. M. 1989, in IAU Symp., 138, ed. J. O. Stenflo, 375

Hathaway, D. H., \& Wilson, R. M. 2004, Sol. phys., 224, 5

Hathaway, D. H., Wilson, R. M., \& Reichmann, J. E. 2002, Sol. Phys., 211, 357
Hiremath, K. M., \& Gokhale, M. H. 1995, ApJ, 448, 437

Hoyt, D. V., \& Schatten, K. H. 1998, Sol. Phys., 181, 491

Knaack, R., \& Stenflo, J. O. 2005, A\&A, 438, 349

Komm, R. W. 1995, Sol. Phys., 156, 17

Mursula, K., Usoskin, I. G., \& Kovaltsov, G. A. 2001, Sol. Phys., 198, 51 Polygiannakis, J. M., Moussas, X., \& Sonett, C. P. 1996, Sol. Phys., 163, 193 Press, W. H., Teukolsky, S. A., Vetterling, W. T., \& Flannery, B. P. 1992, in Numerical Recipes in C (Cambridge University Press), second edition, 640 Pudovkin, M. I., \& Benevolenska, E. E. 1985, Sol. Phys., 95, 381

Rozelot, J. P. 1994, Sol. Phys., 149, 149

Ruzmaikin, A., Feynman, J., \& Robinson, P. 1994, Sol. Phys., 149, 395

Sonett, C. P., \& Trebisky, T. J. 1986, Nature, 322, 615

Stenflo, J. O., \& Vogel, M. 1986, Nature, 319, 285

Tipler, P. A., \& Mosca, G. 2003, in Physics for Scientists and Engineers (New York: Freeman and company), 451

Usoskin, I. G., Mursula, K., Solanki, S., Schssler, M., \& Alanko, K. 2004, A\&A, 413,745

Verdes, P. F., Granitto, P. M., \& Ceccatto, H. A. 2004, Sol. Phys., 221, 167

Waldmeier, M. 1961, in The Sunspot-Activity in the Years 1610-1960, 24 